\title{
DEVELOPMENT OF SYNTHESIS METHOD OF FUNCTIONAL DIAGNOSTIC SYSTEM FOR THRUSTERS OF UNDERWATER VEHICLES
}

\author{
ALEKSANDER ZUEV \\ Institute of Automation and Control Processes FEB RAS, 5, Radio str., \\ Vladivostok, 690041, Russia \\ E-mail:zuev@dvo.ru \\ www.iacp.dvo.ru \\ ALEXEY ZHIRABOK \\ Institute of Marine Technology Problems FEB RAS, 5a, Sukhanov str., \\ Vladivostok, 690091, Russia \\ E-mail: zhirabok@mail.ru \\ www.imtp.febras.ru
}

\begin{abstract}
This paper presents a new synthesis method of functional diagnostic system for thrusters of underwater vehicles (UVs). This system provides detection, localization and identification of minor faults in thrusters of the UV. Proposed method involves two main stages. At the first stage, a bank of diagnostic observers is built to detect and localize the emerging faults. At the same time, each observer is constructed according to a special procedure in such a way as to be sensitive to a different set of the possible faults. At the second stage, the additional observers working in a sliding mode regime are synthesized in order to accurately estimate error values in signals obtained from the UV thruster sensors, and to evaluate deviations of the thruster parameters from their nominal values due to the faults. The results of the simulation show the efficiency and high quality of all synthesized observers.
\end{abstract}

\section{Introduction}

One of the most important tasks arising in the process when the UVs perform various missions is to ensure their safety and fault tolerance. Thrusters of the UVs, that provide their motion along prescribed trajectories, are among the UVs components on which their ability to perform assigned tasks substantially depends. The appearance of any faults that cause a failure or change in the parameters of the thrusters leads to a significant decrease in the performance of the UVs control, various emergency situations, or even the loss of expensive devices.

One of the promising ways to increase the efficiency of the UVs operation is the use of functional diagnostic methods $[1,2]$ for the detection and localization of emerging faults, as well as identification methods [3], which provide an accurate estimation of the error values in the signals received from the UVs onboard sensors, and estimation of the deviations of the UVs thruster parameters from nominal values due to the occurrence of these faults. In the future, the obtained estimates can be used to eliminate the consequences of the emerging faults $[4,5]$ (accommodation to these faults).

At present, in order to assess the technical condition of various elements, the UVs are equipped with alarm control systems whose tasks include identifying the critical and emergency situations $[6,7]$. However, as a rule, the existing systems provide only general monitoring of operability and do not allow to detect the faults, as well as to evaluate errors in the sensors readings and the deviation of the UVs thrusters parameters from nominal values.

Currently, there are several approaches to the construct of the special systems for diagnosing of the UVs thrusters. In particular, the methods based on constructing of the 
observers using the UVs dynamic models are presented in [8-11]. However, since the UVs are described by very complex multiply connected nonlinear differential equations with variable and indefinite parameters, the resulting observers are very complex and do not allow highquality detection and identification of the faults if the UVs carry out motion at high speeds. In addition, many of these methods require the use of special test modes of the UVs movement [11].

It is possible to distinguish the diagnostic methods based on the use of neural networks [12, 13]. The disadvantage of these methods is the necessity for a complex training procedure by using special test motions of the UVs.

In [14], the approach to the construction of the systems of the faults diagnostics and accommodation to these faults in the UVs thrusters was considered. This approach suggests the disconnection of the faulty thruster and the subsequent distribution of its power between the remaining thrusters. The disadvantage of this approach is the fact that UVs must be equipped with an excessive number of the thrusters.

Currently, one of the promising approaches to the detection and identification of the faults is the use of the diagnostic observers operating in the sliding mode regime [15] (the sliding mode observers (SMOs)). Nowadays, the SMOs are applied to solve the problems of the faults identification in linear [16] and nonlinear [17] systems, to ensure the fault-tolerant control [18]. However, in all these works, a number of significant restrictions are imposed on the original system, and besides, the construction of the full-order observers is also assumed. It makes the procedure of the accurate identification of the faults in the UVs thrusters more complicated. In addition, the existing SMOs, as a rule, can be built to detect only single fault.

The conducted analysis shows that due to the high complexity of the implementation of the obtained observers or low accuracy of the identification, most of these methods and approaches cannot be effectively used for the purpose of synthesis the functional diagnostics system for the UVs thrusters.

Thus, the task of developing the new easily implemented and effective method for constructing functional diagnostics systems for the UVs thrusters, providing both detection and localization of the faults, as well as identification of the error values in the signals received from the UVs thruster sensors, and the deviations from the nominal values of the thruster parameters due to the occurrence of these faults, remains unresolved and relevant.

\section{Description of the thruster's model}

We consider the model of the thruster based on a DC motor with angular velocity and current sensors. It is assumed that when the UVs function autonomously, the following typical faults may occur in their thrusters: 1) the fault in the angular velocity sensor, leading to the appearance of the constant or variable error $\widetilde{\omega}(t)$ in its readings; 2 ) motor overheating or shorting of several turns of the armature winding, which leads to a change $\widetilde{R}(t)$ in the nominal value of electrical resistance $R ; 3)$ the appearance of an additional external torque effect $\widetilde{M}(t)$ on the motor shaft, caused, for example, by winding plants on a screw.

The presence of these faults reduces the quality indicators of the thrusters and the accuracy of the movement of the UV along the prescribed paths. In the presence of these faults, the open circuit of each electric motor in matrix form can be described by the following differential equation:

$$
\dot{x}(t)=A x(t)+B u(t)+W(t)+D d(t), \quad y(t)=C x(t)+D_{s} \widetilde{\omega}(t),
$$


where $A=\left[\begin{array}{cc}-k_{v} / J & k_{m} / J \\ -k_{w} / L & -R / L\end{array}\right] ; B=\left[\begin{array}{c}0 \\ k_{u} / L\end{array}\right] ; C=\left[\begin{array}{ll}1 & 0 \\ 0 & 1\end{array}\right] ; W(t)=\left[\begin{array}{c}-M(t) / J \\ 0\end{array}\right] ; \quad D=\left[\begin{array}{cc}1 & 0 \\ 0 & 1\end{array}\right] ;$ $D_{s}=\left[\begin{array}{l}1 \\ 0\end{array}\right] ; \quad x(t)=\left[\begin{array}{c}\omega(t) \\ I(t)\end{array}\right] ; \quad y(t)=\left[\begin{array}{c}\omega(t) \\ I(t)\end{array}\right] ; \quad d(t)=\left[\begin{array}{c}d_{1}(t) \\ d_{2}(t)\end{array}\right] ; \quad d_{1}(t)=-\tilde{M}(t) / J ;$ $d_{2}(t)=-\widetilde{R}(t) I(t) / L ; k_{v}$ is a coefficient of viscous friction; $J$ is a moment of inertia of a rotor of the electric motor, $I(t), L$ are a current and inductance of the armature circuit of the motor, respectively; $k_{w}$ is a coefficient of counter-emf.; $k_{u}$ is a gain of electric amplifier; $k_{m}$ is a torque coefficient; $\omega(t)$ is a rotor angular velocity; $M(t)=\left(k_{1}+k_{2} \lambda+k_{3} \lambda^{2}\right) \rho|\omega(t)| \omega(t) N^{5}$ is a load moment due to the action of a viscous environment on the screw; $\rho$ is density of water; $N$ is a propeller diameter; $\lambda=\eta(t) /(\omega(t) N) ; \eta(t)$ is the UV velocity; $k_{1}, k_{2}, k_{3}$ are known constant coefficients; $u(t)$ is a voltage at an input of a power amplifier.

During the operation of the UVs (especially in stand-alone modes), each fault in any of the thrusters, regardless of the reason for its appearance, should be detected in a timely manner, and its influence on the quality of the thruster work should be eliminated.

Thus, in this paper we pose and solve the task of creating the method for synthesizing the functional diagnostic systems for the UVs thrusters that ensure timely detection and localization of the emerging faults (i.e., determining the fact and time of appearance of specific functions $d_{1}(t), d_{2}(t)$ and $\widetilde{\omega}(t)$ in system (1)), as well as identification of the error values $\widetilde{\omega}(t)$ in the signals received from the angular velocity sensor, and the deviations of the propulsion parameters $\widetilde{R}(t)$ and $\tilde{M}(t)$ from their nominal values due to the appearance of the faults. Moreover, the software implementation of the created system must have minimal computational complexity.

To address this issue, the new method that includes two main stages, is proposed:

1. Construction of the bank of the diagnostic observers (DOs) for the detection and localization of the emerging faults. Moreover, each observer of this bank is built according to a special procedure in such a way as to be sensitive to a different set of functions $d_{1}(t), d_{2}(t)$ and $\widetilde{\omega}(t)$.

2. The construction of the additional SMOs for the purpose to accurately estimate the errors in the signals received from the sensors and the deviations of the UVs thrusters parameters from their nominal values due to the appearance of the faults. In this case, in contrast to existing solutions, it is proposed to use the reduced (having a lower dimension) models of the original system (1) when constructing the indicated observers. This approach permits to reduce the complexity of the SMOs in comparison with the well-known works $[16,18]$, where the observers are constructed in full order. Next, the indicated synthesis steps are considered sequentially.

\section{Building of the DOs for the detection and localization of faults}

For the detection and localization of the emerging faults, the bank of DOs having the following form is built:

$$
\dot{x}_{*}(t)=A_{*} x_{*}(t)+B_{*} u(t)+W_{*}(t)+J_{*} y(t)+K_{*} r(t), \quad y_{*}(t)=C_{*} x_{*}(t),
$$

where $x_{*}$ is the state vector of DO; $r(t)=R_{*} y(t)-y_{*}(t)$ is the output error or residual (in the absence of the faults $r(t)=0$, and when they appear $r(t) \neq 0) ; A_{*}, B_{*}, C_{*}, W_{*}, K_{*}, R_{*}$ и $J_{*}$ are vectors and matrices to be determined; $y_{*}$ is the output vector of the DO.

It is assumed [2] that, in the absence of the faults, there are equalities $x_{*}(t)=\Phi x(t)$ and 
$y_{*}(t)=R_{*} y(t)$ for some matrices $\Phi$ и $R_{*}$, satisfying the following conditions:

$$
\Phi A=A_{*} \Phi+J_{*} C, \quad R_{*} C_{*}=C \Phi, \quad B_{*}=\Phi B, \quad W_{*}(t)=\Phi W(t) .
$$

Next, we consider the procedure for constructing the DOs (2) that are sensitive to a different set of the functions $d_{1}(t), d_{2}(t)$ and $\widetilde{\omega}(t)$. It should be noted that sensitivity to the functions $d_{1}(t)$ and $d_{2}(t)$ is defined by the condition $\Phi D_{j} \neq 0(j=\overline{1,2})$, sensitivity to the errors in the readings of the angular velocity sensors - by condition $J_{*} D_{s} \neq 0$ or $R_{*} D_{s} \neq 0$.

Matrices $A_{*}$ and $C_{*}$, with size of $k \times k$ and $1 \times k$ (where $k$ is the order of DO) are defined in canonical form. In this case, as shown in [2], the Eq. (3) can be reduced to one equation

$$
R_{*} C A^{k}=J_{*_{1}} C A^{k-1}+J_{*_{2}} C A^{k-2}+\ldots+J_{*_{k}} C,
$$

where $J_{*_{i}}-\mathrm{i}$-th row of matrix $J_{*}$.

For the further construction of the DO, it is necessary to determine $B_{*}, W_{*}, J_{*}, \Phi$ and vector $R_{*}$ according to the following new algorithm.

1. Accept $k=1$.

2. Solve Eq. (4). Note that in fact this equation is the system of the linear algebraic equations, while the number of variables can be more than the number of the equations. In this case, some elements of the vector $R_{*}$ and matrix $J_{*}$ will be free variables and can be selected on the basis of the need to ensure the sensitivity or insensitivity to the certain functions $d_{1}(t)$, $d_{2}(t)$ and $\widetilde{\omega}(t)$

If the solution to Eq. (4) does not exist, assume $k:=k+1$ and return to step 1 .

3. Determine the rows of the matrix $\Phi$ by the relations $\Phi_{1}=R_{*} C, \quad \Phi_{i+1}=\Phi_{i} A-J_{*_{i}} C$, $i=1,2, \ldots, k$.

4. Determine the matrix $B_{*}$ and $W_{*}$ by the relations: $B_{*}=\Phi B, \quad W_{*}(t)=\Phi W(t)$.

5. Choose a matrix $K_{*}$ so that $A_{* *}=A_{*}-K_{*} C_{*}$ becomes the stable matrix. Since the matrices $A_{*}$ and $C_{*}$ are found out in canonical form, the $K_{*}$ matrix always exists.

As a result of using the proposed procedure for the UV thruster model (1), the following DOs described below by the matrices were synthesized:

1) First-order $\mathrm{DO}_{1}$, sensitive to $d_{2}(t)$ and $\widetilde{\omega}(t)$ and invariant to $d_{1}(t)$ :

$$
\begin{gathered}
A_{*}=0, \quad C_{*}=1, \quad R_{*}=\Phi=\left[\begin{array}{ll}
0 & 1
\end{array}\right], \quad J_{*}=\left[\begin{array}{ll}
-k_{w} / L & -R / L
\end{array}\right], \\
B_{*}=k_{u} / L, \quad W_{*}(t)=0, \quad K_{*}=100 ;
\end{gathered}
$$

2) First-order $\mathrm{DO}_{2}$, sensitive to $d_{1}(t)$ and $\widetilde{\omega}(t)$ and invariant to $d_{2}(t)$ :

$$
\begin{array}{rll}
A_{*}=0, & C_{*}=1, & R_{*}=\Phi=\left[\begin{array}{ll}
1 & 0
\end{array}\right], \quad B_{*}=0, \\
J_{*}=\left[\begin{array}{ll}
-k_{v} / J & k_{m} / J
\end{array}\right], & W_{*}(t)=-M(t) / J, \quad K_{*}=100 ;
\end{array}
$$

3) $\mathrm{DO}_{3}$ of the second order, sensitive to $d_{1}(t)$ and $d_{2}(t)$ and invariant to $\widetilde{\omega}(t)$ :

$$
\begin{gathered}
A_{*}=\left[\begin{array}{ll}
0 & 1 \\
0 & 0
\end{array}\right], \quad C_{*}=\left[\begin{array}{ll}
1 & 0
\end{array}\right], \quad R_{*}=\left[\begin{array}{ll}
0 & 1
\end{array}\right], \quad J_{*}=\left[\begin{array}{cc}
0 & -\frac{k_{v}}{J}-\frac{R}{L} \\
0 & -\frac{k_{m} k_{w}}{J L}-\frac{k_{v} R}{J L}
\end{array}\right], \quad K_{*}=\left[\begin{array}{l}
200 \\
100
\end{array}\right], \\
B_{*}=\left[\begin{array}{c}
k_{u} / L \\
k_{v} k_{u} / J L
\end{array}\right], \quad \Phi=\left[\begin{array}{cc}
0 & 1 \\
-k_{w} / L & k_{v} / J
\end{array}\right], \quad W_{*}(t)=\left[\begin{array}{c}
0 \\
M(t) k_{w} / J L
\end{array}\right] .
\end{gathered}
$$

The constructed observers $\mathrm{DO}_{1}, \mathrm{DO}_{2}$, and $\mathrm{DO}_{3}$ make it possible to determine the fact and time of the occurrence of the unknown nonzero functions $d_{1}(t), d_{2}(t)$ and $\widetilde{\omega}(t)$ in the system (1). The decision about localization of particular fault is made on the basis of the matrix of 
syndromes $S$, the values of which are the characters 0 and 1 . If the error in the output of the particular DO is sensitive to the particular fault, then at the intersection of the line of the matrix corresponding to this DO and its column corresponding to the detected fault, consist 1 . Otherwise - 0 [2]:

$$
S=\begin{array}{cccc} 
& d_{1}(t) & d_{2}(t) & \widetilde{\omega}(t) \\
r_{1}(t) & 0 & 1 & 1 \\
r_{2}(t) & 1 & 0 & 1 \\
r_{3}(t) & 1 & 1 & 0
\end{array},
$$

where $r_{1}(t), r_{2}(t)$ and $r_{3}(t)$ are residuals, generated by $\mathrm{DO}_{1}, \mathrm{DO}_{2}$ and $\mathrm{DO}_{3}$ correspondingly.

\section{Building of the additional SMOs for fault identification}

It is proposed to carry out the estimation of the errors values in the signals received from the angular velocity sensor and the deviations of the thrusters parameters from their nominal values due to the appearance of the faults using the additional SMOs. Moreover, these observers should be built on the basis of the reduced models [19-21] of the original system (1), which will reduce the complexity of the implementation of the SMOs in comparison with the known works [16, $18]$.

An analysis of the matrix of syndromes $S$ shows that the values of the unknown functions $d_{1}(t)$ and $d_{2}(t)$ can be determined using $\mathrm{DO}_{2}$ and $\mathrm{DO}_{1}$, respectively. Thus, it is advisable to construct the reduced models of the original system, taking these observers as a basis. In general, the obtained first-order models will have the form [20]

$$
\dot{x}_{*}(t)=B_{*} u(t)+J_{*} y(t)+W_{*}(t)+\Phi D d(t), \quad y_{*}(t)=x_{*}(t) .
$$

The SMO for model (8) is

$$
\dot{\hat{x}}_{*}(t)=B_{*} u(t)+J_{*} y(t)+W_{*}(t)+\Phi D v(t)+K_{*} \hat{e}_{y}(t), \quad \hat{y}_{*}(t)=\hat{x}_{*}(t),
$$

where $v(t)=\left\{\begin{array}{cl}g \frac{\hat{e}_{y}(t)}{\left\|\hat{e}_{y}(t)\right\|}, & \text { if } \hat{e}_{y}(t) \neq 0, \\ 0, & \text { if } \hat{e}_{y}(t)=0,\end{array} \hat{e}_{y}(t)=y_{*}(t)-\hat{y}_{*}(t)\right.$.

It was shown in [20] that, when choosing a scalar $g>\|d(t)\|$, the estimation error $\hat{e}(t)=x_{*}(t)-\hat{x}_{*}(t)$ is asymptotically stable, which ensures the existence of the sliding mode, and the value $d(t)$ is estimated with a high degree of accuracy using the following expression: $d(t)=v_{e q}(t)=g \frac{\hat{e}_{y}(t)}{\left\|\hat{e}_{y}(t)\right\|+\delta}$, where $\delta$ is small positive constant [16].

Thus, taking into account (5) and (6), the following SOs were obtained:

1) $\mathrm{SMO}_{1}$ for identification of the value $\tilde{M}(t)$ :

$$
\begin{gathered}
\dot{\hat{x}}_{*}(t)=-\frac{k_{v}}{J} y_{1}(t)+\frac{k_{m}}{J} y_{2}(t)-\frac{M(t)}{J}+0.1 \hat{e}_{y}(t)+v_{e q}(t), \quad \hat{y}_{*}(t)=\hat{x}_{*}(t), \\
\hat{e}_{y}(t)=y_{1}(t)-\hat{y}_{*}(t), \quad g=100, \quad \delta=0.01, \quad \tilde{M}(t)=-v_{e q}(t) J
\end{gathered}
$$

2) $\mathrm{SMO}_{2}$ for identification of the value $\widetilde{R}(t)$ : 


$$
\begin{gathered}
\dot{\hat{x}}_{*}(t)=-\frac{k_{w}}{L} y_{1}(t)-\frac{R}{L} y_{2}(t)+\frac{k_{u}}{L} u(t)+0.1 \hat{e}_{y}(t)+v_{e q}(t), \quad \hat{y}_{*}(t)=\hat{x}_{*}(t), \\
\hat{e}_{y}(t)=y_{2}(t)-\hat{y}_{*}(t), \quad g=5000, \quad \delta=1, \quad \widetilde{R}(t)=-v_{e q}(t) L / I(t) ;
\end{gathered}
$$

The identification of the value of the function $\widetilde{\omega}(t)$, and consequently, the estimation of the error in the readings of the angular velocity sensor of the thruster of UV can be carried out using the following reduced model [21], built on the basis of $\mathrm{DO}_{1}$ :

$$
\dot{x}_{*}(t)=B_{*} u(t)+J_{*} y(t)+W_{*}(t)-J_{*} D_{s} \widetilde{\omega}(t), \quad y_{*}(t)=x_{*}(t) .
$$

By analogy with other SMOs, the $\mathrm{SMO}_{3}$ based on the model (12) for the estimation of the value $\widetilde{\omega}(t)$ has the following form:

$$
\begin{gathered}
\dot{\hat{x}}_{*}(t)=-\frac{k_{w}}{L} y_{1}(t)-\frac{R}{L} y_{2}(t)+\frac{k_{u}}{L} u(t)+0.1 \hat{e}_{y}(t)+\frac{k_{w}}{L} v_{e q}(t), \quad \hat{y}_{*}(t)=\hat{x}_{*}(t), \\
\hat{e}_{y}(t)=y_{2}(t)-\hat{y}_{*}(t), \quad g=1, \quad \delta=1, \quad \widetilde{\omega}(t)=v_{e q}(t) .
\end{gathered}
$$

Thus, due to the use of the $\mathrm{SMO}_{1}, \mathrm{SMO}_{2}$ and $\mathrm{SMO}_{3}$, it is possible to provide the estimation of the errors in the signals received from the sensors and the deviations of the thruster parameters from their nominal values due to the appearance of the faults. It is important to note that the use of the reduced models (8) and (12) made it possible to construct the simple first-order observers. The structural diagram of the synthesized fault diagnosis system for the UV thrusters is shown in Figure 1.

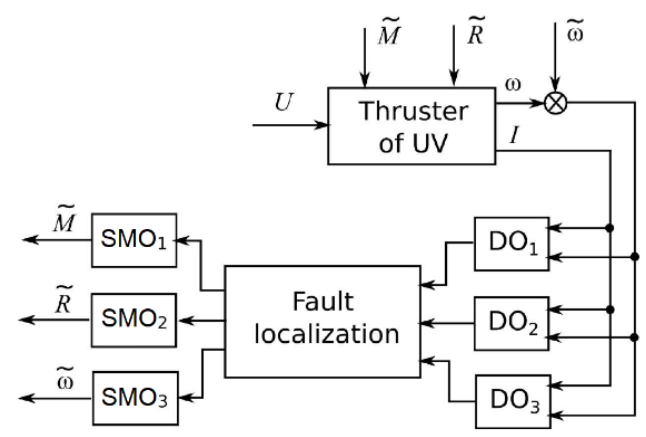

Figure 1. System of fault diagnosis of the UV thrusters.

\section{Simulation results}

To test the operability and effectiveness of the proposed synthesis method, the operation of the functional diagnostics system of the UV thrusters was simulated by MATLAB/Simulink. At simulation the following parameters of the UV thruster with DC motor FL42BLSH03 were used: $k_{v}=67.5610 \cdot 10^{-5} \mathrm{Nms} / \mathrm{rad} ; \quad J=0,025 \mathrm{kgm}^{2} ; R=0.65 \Omega ; L=0.26 \mathrm{mH}$; $k_{w}=0.166 \mathrm{Vs} / \mathrm{rad} ; k_{u}=27.7 ; k_{m}=0.169 \mathrm{Nm} / \mathrm{A} ; D=0.122 \mathrm{~m} ; k_{1}=0.0167 ; k_{2}=-0.0159$; $k_{3}=-0.0047$. For simplicity, we considered mooring mode, when $\eta(t)=0$. This assumption did not reduce the significance of the study of the performance of diagnostic observers, but excluded the need for a very complex modeling of the dynamics of the UV.

The thruster was controlled by input $u(t)=1,5+\sin (t)$, and single faults were simulated by introducing the external toque $\tilde{M}(t)=0.2 \sin ((t-3) \pi / 4) \mathrm{Nm}$ in the period of time $3 \div 7 \mathrm{~s}$; a smooth change in the value of active resistance $0.1 \Omega$ in the period of $5 \div 10 \mathrm{~s}$; introduction of the constant error $\widetilde{\omega}(t)=0.2 \mathrm{rad} / \mathrm{s}$ in the readings of the speed sensor for a period of $4 \div 10 \mathrm{~s}$. 
Figure 2 presents graphs of the function $\tilde{M}(t)$ (curve 1) and its estimations (curve 2) using the proposed observers. Figure 3 and 4 show similar graphs for the functions $\widetilde{R}(t)$ and $\widetilde{\omega}(t)$. From the presented figures it can be seen that the constructed observers allow to determine the time of the appearance of the errors, and also provide the fairly accurate estimation of the magnitude of these errors. The identification error in all three cases did not exceed $0.1 \%$.

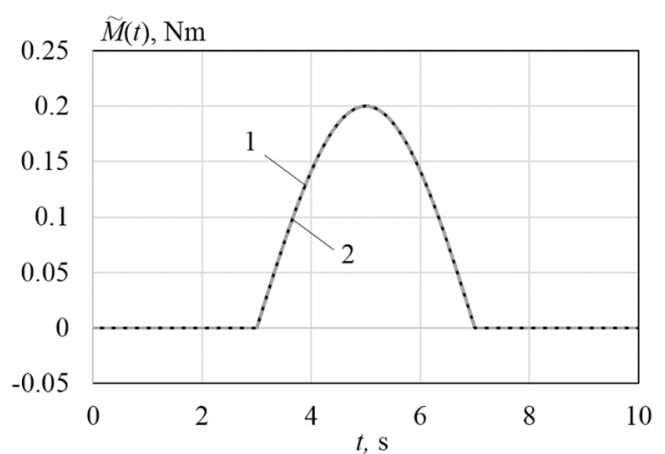

Figure 2. Function $\tilde{M}(t)$ (curve 1) and its estimate (curve 2).

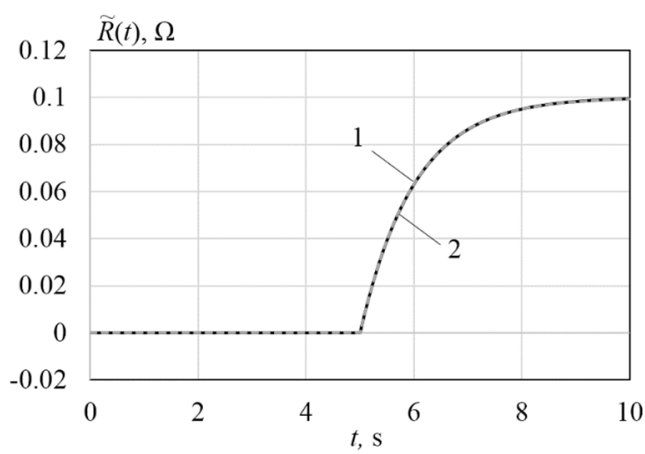

Figure 3. Function $\widetilde{R}(t)$ (curve 1) and its estimate (curve 2).

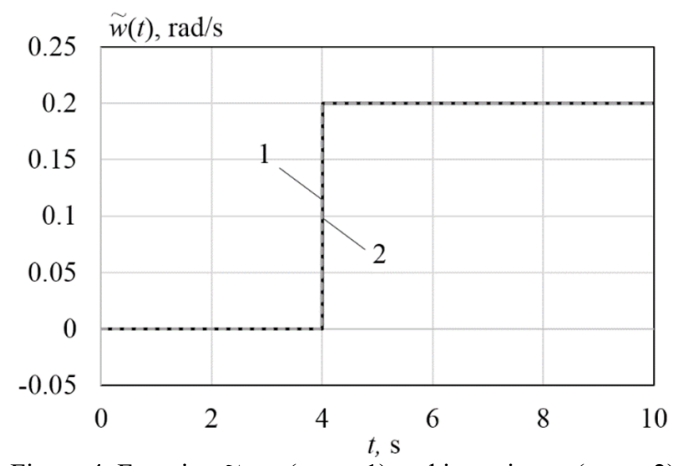

Figure 4. Function $\widetilde{\omega}(t)$ (curve 1 ) and its estimate (curve 2).

Thus, the results of the studies show the unconditional efficiency and high quality of the synthesized observers. In all the cases considered, it was possible to timely detect the fact of the appearance of the fault, as well as provide the determination of its value. Based on the considered approach to the construction of diagnostics systems for the UVs thrusters, highly reliable UVs control systems can be created.

\section{Conclusion}

Summarizing the above, we can draw the following conclusions. The systems of functional diagnostics of the UVs thrusters synthesized using the two-stage method proposed in the work are quite simple and have low computational complexity, that allows them to be implemented on typical on-board computers of the UVs.

The constructed systems provide not only the timely detection and localization of the arising typical faults with the help of the bank of the DOs, but also the accurate estimation of the errors in the signals received from the sensors and the deviations of the thrusters parameters from their nominal values due to the appearance of these faults. The simulation results confirm the efficiency and high quality of the synthesized observers.

\section{Acknowledgments}

This research was supported by Russian Science Foundation (project № 18-79-00143). 


\section{References}

1. P. Frank, "Fault diagnosis in dynamic systems using analytical and knowledge-based redundancy. A survey and some new results", Automatica. 26 (1990), pp. 459-474.

2. A. Zhirabok, A. Shumsky, S. Solyanik, A. Suvorov, "Fault detection in nonlinear systems via linear methods", Int. J. Applied Mathematics and Computer Science. 27 (2017), pp. 261-272.

3. S. Simani, C. Fantuzzi, R. Patton, Model-based Fault Diagnosis in Dynamic Systems Using Identification, Berlin. Heidelberg: Springer-Verlag. (2002).

4. M. Blanke, M. Kinnaert, J. Lunze, M. Staroswiecki, Diagnosis and Fault-Tolerant Control, Berlin: Springer-Verlag. (2006).

5. V. Filaretov, A. Zhirabok, A. Zuev, A. Protcenko, "The development of the faults accommodation system for actuators of multilink manipulators", Proc. of the 23rd DAAAM Int. Symposium on Intelligent Manufacturing and Automation. (2012), pp. 575-578.

6. G. Chirikjian, Robotic Self-replication, Self-diagnosis, and Self-repair: Probabilistic Considerations, Distributed Autonomous Robotic Systems. (2009), pp. -273-281.

7. A. Pisarets, A. Zhirabok, A. Inzartsev, "On diagnosis for thrusters of underwater vehicles", Proc. of the Sixth ISOPE Pacific/Asia Offshore Mechanics Symposium. (2004), pp. 255259.

8. M. Zhang, J. Wu, Y. Wang, "Simultaneous Faults Detection and location of Thrusters and Sensors for Autonomous Underwater Vehicle", Proc. of the Fourth International Conference on Intelligent Computation Technology and Automation. (2011), pp. 504-507.

9. D. Zhu, B. Sun, "Information fusion fault diagnosis method for unmanned underwater vehicle thrusters", IET Electrical Systems in Transportation. 3 (2013), pp. 102-111.

10. J. Wang "Fault Diagnosis of Underwater Vehicle with FNN", Proc. of the 10th World Congress on Intelligent Control and Automation. (2012), pp. 2931-2934.

11. B. Zhao, R. Skjetne, M. Blanke, F. Dukan, "Particle Filter for Fault Diagnosis and Robust Navigation of Underwater Robot”, IEEE Transactions on Control Systems Technology. 22 (2014), pp. 2399-2407.

12. J. Wang, G. Wu, L. Wan, Y. Sun, D. Jiang, "Recurrent Neural Network Applied to Fault Diagnosis of Underwater Robots", Proc. of the IEEE International Conference on Intelligent Computing and Intelligent Systems. (2009), pp. 593-598.

13. J. Wang, "Fault Diagnosis of Underwater Vehicle with Neural Network", Proc. of the 24th Chinese Control and Decision Conference (CCDC). (2012), pp. 1613-1617.

14. N. Sarkar, T. Podder, G. Antonelli, "Fault-Accommodating Thruster Force Allocation of an AUV Considering Thruster Redundancy and Saturation", IEEE Transactions on Robotics and Automation, 8 (2002), pp. 223 - 233.

15. V. Utkin, Sliding Modes in Control Optimization, Berlin: Springer, (1992).

16. C. Edwards, S. Spurgeon, R. Patton, "Sliding Mode Observers for Fault Detection and Isolation", Automatica, 36 (2000), pp. 541-553.

17. J. He, C. Zhang, "Fault Reconstruction Based on Sliding Mode Observer for Nonlinear Systems", Mathematical Problems in Engineering, (2012), pp. 1-22.

18. H. Alwi, C. Edwards, "Fault Tolerant Control Using Sliding Modes with On-line Control Allocation", Automatica, 44 (2008), pp. 1859-1866.

19. A. Zhirabok, A. Zuev V., A. Shumskii, "Diagnosis of Linear Systems Based on Sliding Mode Observers", Journal of Computer and Systems Sciences International, 58 (2019), pp. 898-914.

20. A. Zhirabok, A. Zuev V., A. Shumskii, "Diagnosis of Linear Dynamic Systems: An Approach Based on Sliding Mode Observers", Automation and Remote Control, 81 (2020), pp. $345-358$.

21. A. Zhirabok, A. Zuev V., A. Shumskii, "Identification of faults in the sensors of technical systems with the use of sliding mode observers", Measurement Techniques, 62 (2020), pp. 869-878. 